\title{
Real time FPGA implemnation of SAR radar reconstruction system based on adaptive OMP compressive sensing
}

\author{
Eslam Ashraf ${ }^{1}$, Ashraf A. M. Khalaf ${ }^{2}$, Sara M. Hassan ${ }^{3}$ \\ ${ }^{1,2}$ Electronics and Communications Engineering Department, Faculty of Engineering Minia University, Egypt \\ ${ }^{3}$ Electronics and Communications Engineering Department, Modern Academy for Engineering and Technology, Egypt
}

\begin{tabular}{|c|c|}
\hline Article Info & ABSTRACT \\
\hline & \multirow{11}{*}{$\begin{array}{l}\text { Synthetic Aperture Radar (SAR) is an imaging system based on the } \\
\text { processing of radar echoes. The produced images have a huge amount of data } \\
\text { which will be stored onboard or transmitted as a digital signal to the ground } \\
\text { station via downlink to be processed. Therefore, some methods of } \\
\text { compression on the raw images provides an attractive option for SAR } \\
\text { systems design. One of these techniques which used for image reconstruction } \\
\text { is the Orthogonal Matching Pursuit (OMP). OMP is an iterative algorithm } \\
\text { which need high computational operations. The computational complexity of } \\
\text { the iterative algorithms is high due to updating operations of the } \\
\text { measurement vector and large number of iterations that are used to } \\
\text { reconstruct the images successfully. This paper presents a new adaptive OMP } \\
\text { algorithm to overcome this issue by using certain threshold. The new } \\
\text { adaptive OMP algorithm is compared with the classical OMP algorithm } \\
\text { using the Receiver Operating Characteristic (ROC) curves. The MATLAB } \\
\text { simulations show that the new adaptive OMP algorithm improves the } \\
\text { probability of detection at lower SNRs, reduce the computational operations } \\
\text { as well as the number of required iterations. FPGA implementation of both } \\
\text { the classical OMP and the adaptive OMP algorithm are also presented in } \\
\text { this paper. }\end{array}$} \\
\hline Received Jan 18, 2020 & \\
\hline Revised Apr 19, 2020 & \\
\hline Accepted May 21, 2020 & \\
\hline Keywords: & \\
\hline Adaptive & \\
\hline Compression & \\
\hline Compressive sensing & \\
\hline OMP & \\
\hline Reconstruction & \\
\hline SAR & \\
\hline
\end{tabular}

Copyright $(9) 2020$ Institute of Advanced Engineering and Science. All rights reserved.

\section{Corresponding Author:}

Eslam Ashraf,

Electronics and Communications Department,

Faculty of Engineering Minia University, Minia, Egypt.

Email: eng.eslamashraff@gmail.com

\section{INTRODUCTION}

The Synthetic Aperture Radar (SAR) has wide applications in the remote sensing field and the mapping. SAR applications provides terrain structural information to geologists, oil spill boundaries on water to environmentalists, and ice hazard maps to navigators, as well as targeting monitoring and tracking for military applications. The parameters of a SAR depend on the requirements to achieve the mission and are determined by the system engineer and the customer $[1,2]$.

SAR performance depends on the capability of multiple imaging modes and resolutions [3]. These features and the wide swath requirement provide a huge amount of raw data that will be processed to generate high resolution suitable images. The low computational resources of the platforms lead to not be able to process data onboard. Thus, it must be stored or transmitted to the ground stations where the processing is performed. The huge amount of raw data produced by the Image Formation Processor (IFP) is constrained by onboard storage and transmission links capabilities. Accordingly, many efforts were achieved to develop a suitable compression technique for compressing the SAR Raw data image [4].

The compression of the SAR raw data is not easy task due to the noise-like characteristics. The noise-like characteristics arises because the signals from several scatters are added incoherently with unknown phase and amplitude [5]. Due to the poor correlation of SAR data, it is not useful to apply the 
compression using redundancy reduction techniques directly $[2,6]$. There are some differences between the SAR images and the optical images, the SAR image is larger in size. Also, its entropy is higher than the entropy of optical image; additionally the SAR image has information in the low frequency bands and high frequency bands, but the optical image has information in the low frequency bands with noise in a high frequency band. Finally, SAR image has a larger dynamic range compared to the optical image. Because of these differences, the SAR needs special techniques for compression that lead the researchers to make excessive efforts to overcome on these issues [7-9].

One of these techniques is the discrete cosine transform (DCT). The compression is achieved by transforming the images to another domain which is done by using the uncorrelated coefficients as some terms can be quantized to zero [10]. However, this technique is not suitable for SAR systems because of the high noise, the high frequency, the dynamic range, and the block of some artifacts in SAR images [11, 12].

Discrete Wavelet Transform (DWT) is a common technique that is used for the image compression; it is based on using a filter bank connected by a down sampling procedure. DWT is better than the DCT, because it can be used in the frequency domain and time domains, it presents a less dynamic range and provides a built-in despeckling noise $[13,14]$. Yet, this technique is not suitable for some systems due to the high computational operations that lead to a high complex system and high consumption of both the power and the resources $[15,16]$.

Because of the previous drawbacks that were presented in the related prior work, there are other methods that were presented to be suitable for the SAR usage. One of these methods is the compressive sensing (CS). CS is a new algorithm that presents a suitable method for the compressing and reconstruction of SAR raw data image [8]. It allows simple schemes unlike the Classical methods [17]. CS enables a large reduction in the sampling and computation costs for data that have compressible representation which is one of the SAR features [18]. There are three types of the compressive sensing algorithms which are: Complex Approximate Message Passing (CAMP), Orthogonal Matching Pursuit (OMP) and $l_{1}$-regularized Least Square method [19]. In this research, we will use the OMP algorithm because it is more accurate in comparison to the CAMP and simpler than the $l_{1}$-regularized Least Square.

In [20], the author presented some algorithms for the sparse signal reconstruction using a noisy estimation measurements, such as the convex optimization algorithms these algorithms need very few measurements however it require a complex computational operations. Another algorithm that was presented in this thesis is the Greedy algorithms which are in some sense a good compromise between those extremes concerning computational complexity and the required number of measurements. Threshold algorithms that require less computational complexity consist of the Hard Threshold (HT) algorithm and The Soft Threshold (ST) algorithm.

In [21], the authors proved that the CAMP algorithm and the separate detector combination achieve better performance. To obtain as adaptive detection scheme, the paper presented a combination from the CAMP algorithm and conventional CFAR processing. In [22], the authors proposes a new adaptive CAMP algorithm based on signal threshold in order to solve the computational complexity shortcoming and improve the detection performance at low signal to noise ratios (SNRs).

In [16], the authors defined the compressive sensing algorithms and proposed a pipelined processing method for one of the CS algorithms to reduce the consumed time using FPGA implementation. Although, the Proposed method reduced the consumed time, the compression and the reconstruction process need a high computational complexity operations.

The well known OMP algorithm performs many iterations and these iterations have a high computational complexity used to updating the residue, in each iteration it requires some matrices mathematical operations in order to reconstruct the image [23]. In this paper, a proposed modification to OMP algorithm is presented by changing the method of calculating the threshold in the reconstruction algorithm, the computational complexity and then the performance of the OMP algorithm are improved. Additionally, the adaptive method, that enables us for reconstructing the image with one iteration, is also used to enhance the performance of the reconstruction. Hence, updating the residue will be eliminated. The proposed algorithm in this article is called adaptive OMP algorithm.

This paper is organized as follows: Section 2 describes OMP algorithm and its steps, Section 3 show the proposed algorithm. Section 4 discusses the hardware implementation, then the simulation and results are presented in Section 5. Finally, the conclusion is explained in Section 6.

\section{THE ORTHOGONAL MATCHING PURSUIT}

The compressive sensing is a reconstruction technique which using under sampled data for reconstructing the images. It is an inverse process that enables of recovering an image using a few measurements. The challenge is to estimate the unknown image using lesser number of given observations [24]. 
In fact, the compressive sensing is a reconstruction method that can be used in the image recovering process. The compression process is an easy task that can be performed by multiplying the sensing matrix $(\Phi)$ with the image coefficients $(\mathrm{X})$ that yields a vector $(y=\Phi \mathrm{X})$ with smaller dimensions. Figure 1 shows Example of sparse image $[25,26]$.

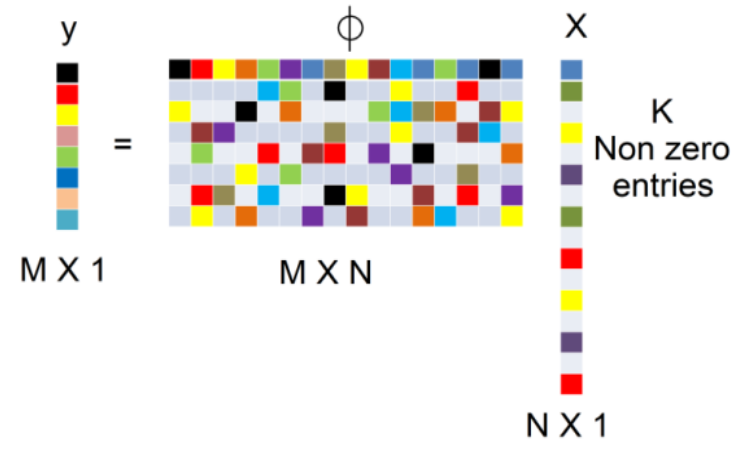

Figure 1. Example of sparse signal

CS demands the measurement matrix to be incoherent with the sparsity basis. Furthermore, the measurement matrix $\Phi$ should satisfy the restricted isometry property [1]. To any k-sparse signal $v, \Phi$ should satisfy $[27,28]$.

\subsection{Incoherence of a matrix}

The coherence property $\mu$ of a matrix can be defined as the smallest angle between each two columns (the largest absolute inner product between any two columns of A). The maximum value of the coherence of any matrix equal 1 in the case that two columns coincide [29].

$$
\mu(A)=\max _{1<i<j \leq N} \frac{\mid\left\langle A_{i} \cdot A_{j}\right\rangle}{\left\|A_{i}\right\|_{2}\left\|A_{j}\right\|_{2}}
$$

The incoherence property is used to make sure that the matrix is sparse matrix. This can be found by designing it to make each column to be orthonormal, and by determining the number of measurements $\mathrm{M}$ by

$$
\mathrm{M} \geq \mathrm{k}^{2} \ln \mathrm{N}
$$

Where $\mathrm{N}$ is the number of Nyquist rate samples, $\mathrm{M}$ is the number of measurements and $\mathrm{k}$ is the number of the non-zero coefficients. If we assume that the received image has a length of 100 samples in other words $\mathrm{N}=100$ and $\mathrm{M}=74$. We will find that the maximum number of $\mathrm{k}$ will be four samples according to (2). If the number of $\mathrm{k}$ exceeds four samples; then, the number of measurements will be larger than the length of the received image so the CS theory will not be effective [30-32].

\subsection{Restricted isometry property}

The Restricted Isometry Property (RIP) is used to handle two types of errors which are the receiver noise that induced inside itself and the reconstruction process error. Because of these errors, it is not possible to guarantee uniqueness which is controlled by the RIP property, which ensures that the recovery process is stable in presence of the noise to control the tolerant for both types of errors the equation that describes the RIP is given by [15-33]:

$$
(1-\gamma)\|x\|_{2}^{2} \leq\|A \cdot x\|_{2}^{2} \leq(1+\gamma)\|x\|_{2}^{2}
$$

Where $\gamma$ is a fixed number less than unity. This condition implies that any $\mathrm{k} \times \mathrm{k}$ sub-matrix of the original matrix $\mathrm{A}$ is near isometry and does not change the norm of a vector considerably. However, for random matrices that are of a particular interest in CS, incoherence and RIP properties present useful performance guarantees [34-36]. 
Consider the measurement vector $\mathrm{y}_{\mathrm{j}}=\{\mathrm{j}=1$ to $\mathrm{M}\}$ as given as:

$$
\begin{aligned}
& \mathrm{y}=\Phi \mathrm{X} \\
& \Phi=\mathrm{R}^{\mathrm{MXN}}
\end{aligned}
$$

Where $\Phi$ is the measurement matrix and $\mathrm{M}<<\mathrm{N}$. Measurement matrix multiplies $\mathrm{x}$ to compress the $\mathrm{X}$ matrix to y matrix; so, each column of $\Phi$ is seen as a sensing column. The original image can be reconstructed by using the OMP algorithm. The steps for performing the OMP algorithm are given as following [17]. Figure 2

Figure 2 shows the flowchart of the OMP compressive sensing.

a) Initialize the residual $r_{0}=y$, the index set $A_{0}=\Phi$ and the iteration count $\mathrm{k}=0$;

b) Choose the index $K_{\mathrm{k}}=\operatorname{argmax}_{\mathrm{i}=1 \ldots \ldots . . \mathrm{M}}\left|<\mathrm{r}_{\mathrm{k}-1} . \Phi_{\mathrm{i}}>\right|$. In case of multiple indices, maximum occurs then break the tie deterministically.

c) Increase the set of index $A_{k}=A_{k-1} v\left\{\Lambda_{k}\right\}$.

d) Solve the least square problem $x_{k}=\operatorname{argmin}\left\|y-\Phi x_{k}\right\|_{2}$, to obtain the latest approximation of the image [37].

e) Notice the new estimate of the data then calculate the new residual: $a_{k}=\Phi_{k} x_{k} \cdot r_{k=} x-a_{k}$.

f) Increment the iteration count $\mathrm{k}$ then return to step 2 if $\mathrm{k}<\mathrm{Q}, \mathrm{Q}$ is the sparsity level [22].

g) The approximation coefficients for the image have nonzero indices at the elements listed in $A_{k}[5,8,18,38]$.

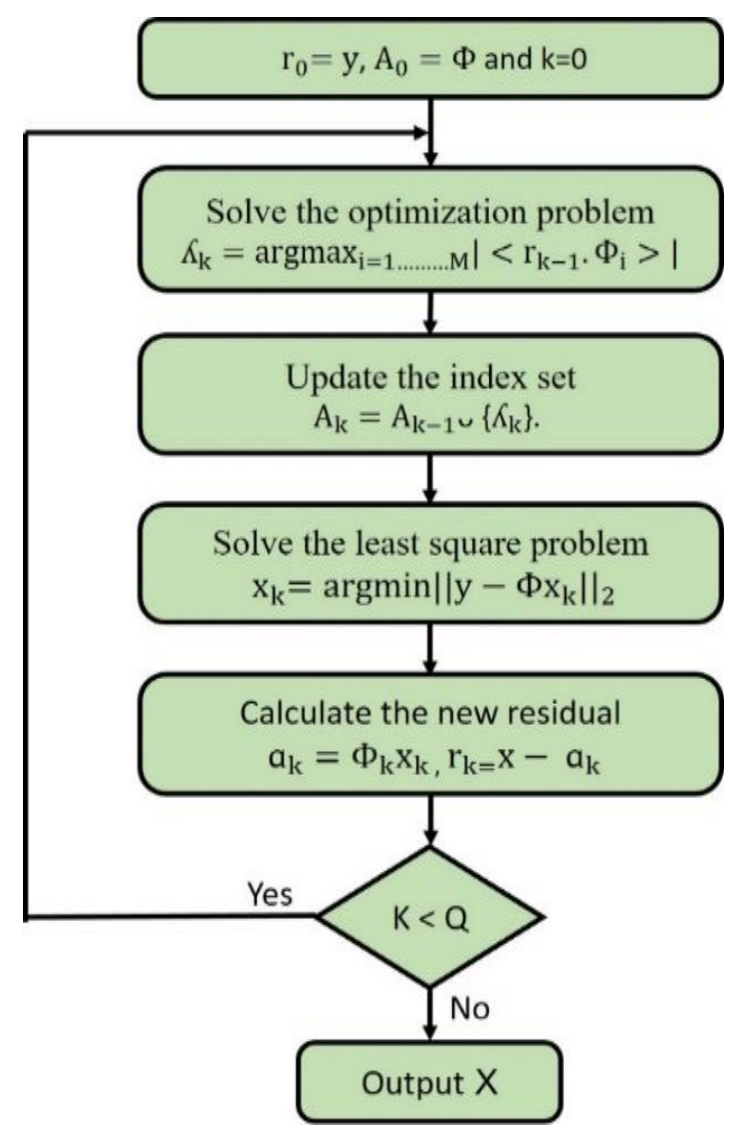

Figure 2. The flowchart of the OMP compressive sensing

\section{PROPOSED Pipelined Algorthim}

A proposed algorithm based on calculating a certain threshold to reconstruct any SAR images is presented here. In this technique, the sensing matrix $\Phi$ is generated to be suitable for the incoherence and restricted isometry property which are discussed before. The sensing matrix $\Phi$ is generated by using the Matlab program to satisfy the two previous discussed properties. The proposed Adaptive OMP algorithm is 
less complex than the OMP algorithm and needs a fewer mathematical operations because it recovers the image with one iteration; hence, it does not need to update the residue.

As mentioned before the vector $y$ is calculated by multiplying the sensing matrix $\Phi$ by the sparse image. Then, the suggested adaptive OMP algorithm is used to recover it by using the measurement vector in three steps; the first step is estimating the noise by using the transpose of the sensing matrix A and the measurement vector $y$. The second step is finding the threshold adaptively based on the absolute noise value the signal $\tilde{x}$. The last step is to refine the estimated signal by the adaptive soft thresholding function. Figure 3 shows the flowchart of the adaptive OMP.

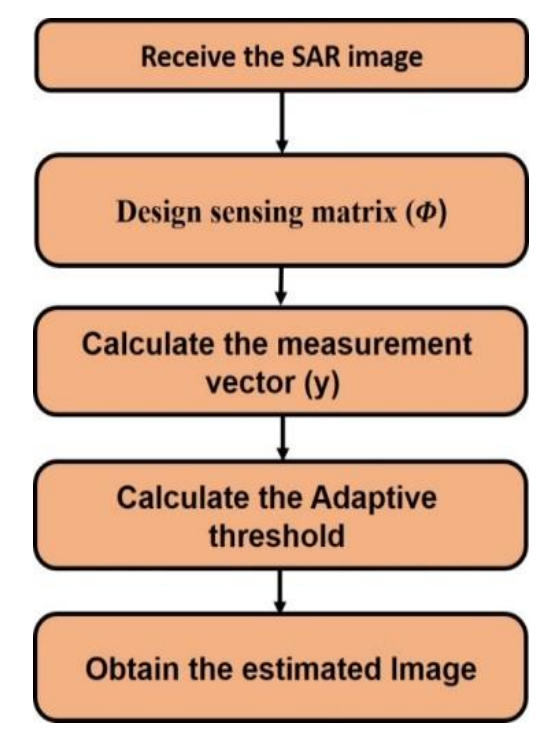

Figure 3. The flowchart of the adaptive OMP

The evaluation of the performance of both the OMP and the proposed adaptive OMP is performed by finding the probability of detection at different SNRs which is called the Receiver Operating Characteristic (ROC) curves as shown in Figure 10 [15, 33]. A comparison between the original OMP algorithm and the adaptive OMP algorithm is presented to provide a clear understanding is obtained. Also, to approve the proposed algorithm, in addition to the mean square error is calculated (the square of difference between the original image and the reconstructed one) as shown in Figure 9.

\section{HARDWARE IMPLEMENTATION}

The normal OMP and the adaptive OMP algorithms are implemented to compare between the two algorithms. The design is implemented by using Xilinx Spartan 3E FPGA starter kit (XC3S1000E-4C in VQ100 package), the project is achieved by VHDL code by using Xilinx ISE14.7 program and it is simulated by using the Modelsim simulator program.

The schematic diagram of the compression part consists of two modules (the compression module and the memory module). These two modules are achieved by writing a VHDL code. Because of the huge amount of the data and to avoid losing any of the received data, the output is saved in a memory to be used in the reconstruction process.

Figure 4 shows the schematic diagram of the implemented CS SAR data compression. The implemented CS SAR data compression is generated by going through synthesize, place and route steps. Synthesize takes the compiled source and produces a digital logic equivalent for the written VHDL code. The logic produced is optimized for the target device and for the constraints applied by the designer. Place and route take all the synthesized logic and connect it inside the FPGA. All the I/O pins are also connected to the corresponding I/O cells and these. In turn, are connected to the required logic cells. The size of each coefficient is 24 bits and each element of the sensing matrix is 8 bits so each output coefficient will be 32 bits. The schematic diagram of the compression using ISE 14.7 is shown in Figure 4. 


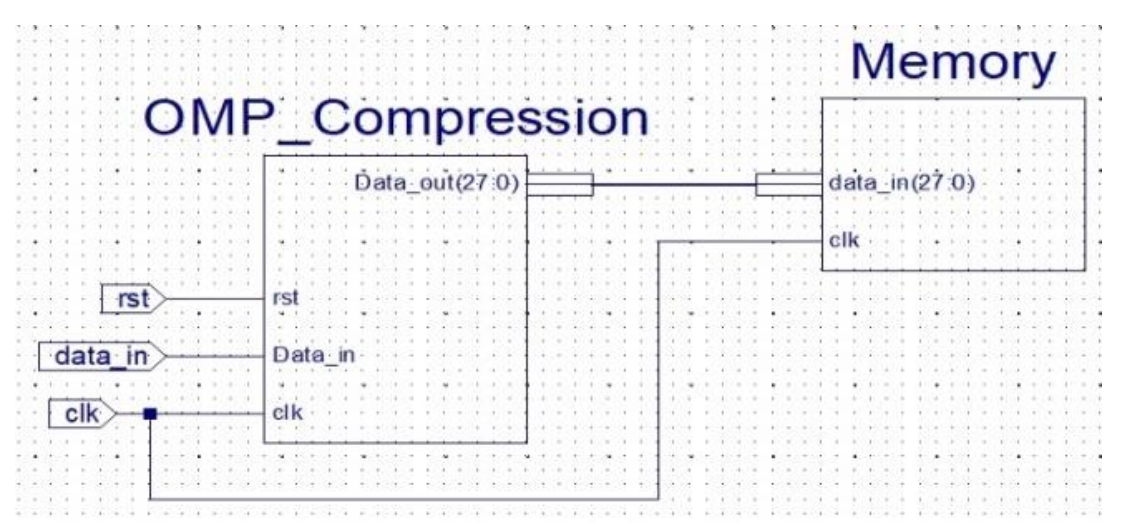

Figure 4. The schematic diagram of the compression

The memory module is used in real time implementation in order to avoid losing any of the received data. The SAR data processing is considered to be processed a in real-time implementation, if all the received data is processed without losing any of the received data or the received data is processed before receiving another data. The received SAR data image $64 \times 256$ pixel complex image which is converted into one dimensional matrix with 16384 image coefficients, these coefficients will be stored in the memory after the compression stage. The serial to the parallel module will be collected every 32 for each coefficient and pass these bits to the adaptive OMP reconstruction module and after receiving all the coefficients, the reconstruction will be performed. The input of the reconstruction module will be synchronized with the output of the serial to parallel module. Figure 5 shows the schematic diagram of the adaptive OMP reconstruction. The Modelsim simulations and the results of the adaptive OMP are shown in Figures 11 and 12, the improved performance will appear after the reconstruction and reviewing the image using Matlab simulation program.

Figure 6 shows the original images that we used to prove our concept which is converted first to numerical coefficients using Matlab simulation program then was compressed using our FPGA implementation. Figure 7 shows the same image after the reconstruction process that was converted from numerical coefficients to image using Matlab after being passed by the compression and the reconstruction using the Classical OMP and the adaptive OMP. As seen in Figure 8, the adaptive OMP will produce improved performance compared to the Classical one. The reconstructed image that was decompressed by using the adaptive OMP is similar to the original one more than the image that was reconstructed using the Classical OMP which approves our concept and approves the proposed algorithm.

The proposed process needs less resources than the Classical one, as appeared in the device utilization summary for the implementation of both the Classical algorithm and the adaptive algorithm. These resources consumption was predicted because the adaptive algorithm use less iteration than the Classical algorithm. As shown in Table 1 and Table 2. The improved performance is presented using the ChipScope (Xilinx ChipScope tool provides virtual I/O directly and logic analyzer to check the internal signals) to check the design after downloading the project on the used FPGA kit to check the behavior. The ChipScope signals are shown in Figure 13 and 14.

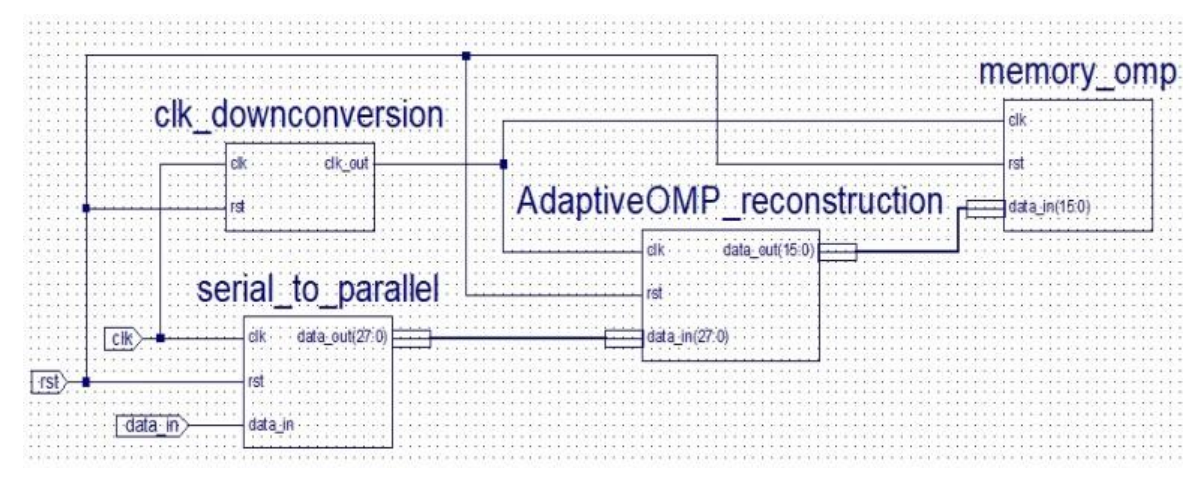

Figure 5. The schematic diagram of the adaptive OMP reconstruction 


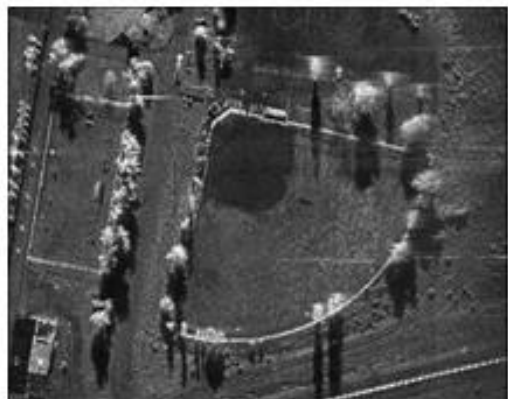

(a)

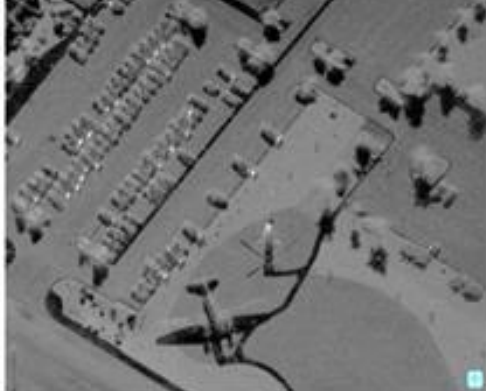

(b)

Figure 6. The original images

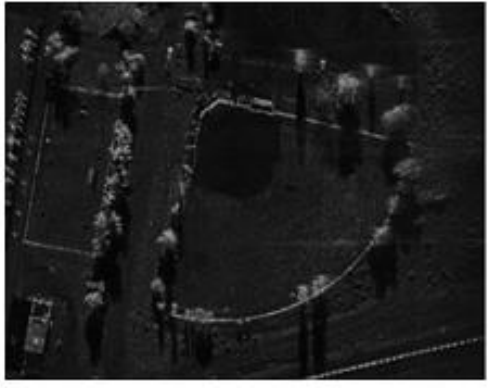

(a)

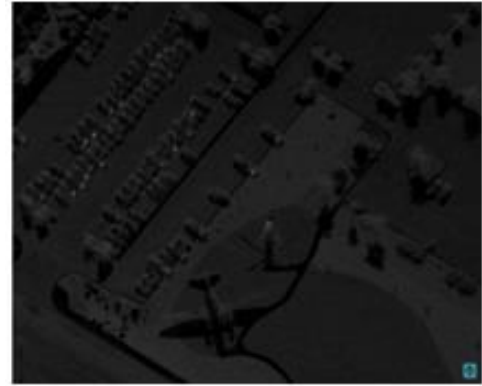

(b)

Figure 7. The reconstruction using the classical OMP

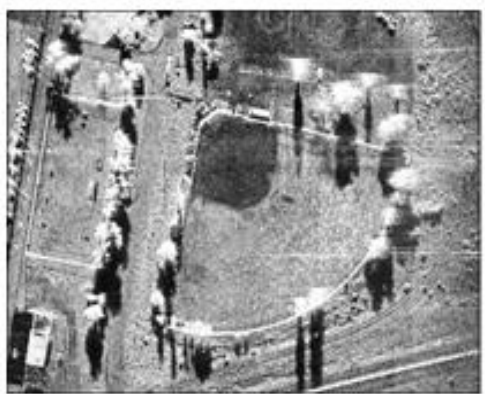

(a)

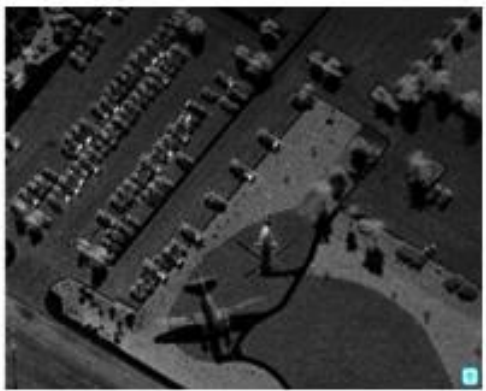

(b)

Figure 8. The Reconstruction using the Adaptive OMP

\section{SIMULATION AND RESULTS}

Figure 9 shows the MATLAB Simulation results of the MSE of reconstructing the image using the Classical and the adaptive OMP algorithms. As shown in Figure 9, the MSE accurately predicts the behavior of the classical and adaptive OMP algorithm. The MSE of the classical OMP algorithm decreases every iteration until it tends to tolerance which is calculated according to the RIP. On the other hand, the MSE of the adaptive OMP algorithm is a constant value tends to zero all the iteration values. Figure 10 shows the simulation results of ROC curves for reconstructing image using the Classical OMP algorithm and the adaptive OMP algorithm. As shown in Figure 10, the adaptive OMP gives a probability of detection value higher than the Classical OMP.

Figure 11 shows the Modelsim simulation of the compression, the coefficients before the compression are being appeared at the left side of the image that consist of 32 bits but the coefficients after the compression are being appeared at the right side of the image which consist of 24 bits. Furthermore, Figure 12 shows the adaptive OMP reconstruction, the coefficients before the reconstruction appear at the left side of the image that consists of 24 bits. However, the coefficients after the reconstruction appear at the right

Real time FPGA implemnation of SAR radar reconstruction system based on adaptive ... (Eslam Ashraf) 
side of the image which consists of 32 bits. The compression ratio (the ratio between the bit size before the compression and the bit size after the compression) will be calculated. This value is important to determine how this algorthim can be effective in the data size reduction. According to the bit size of the coefficients that are shown in Figrure 11 and Figure 12, the original coefficient consists of 32 bits and the compressed coefficient consists of 24 bits so the compression ratio will equal 1.3. This compression ratio means that the size of the compressed image will be reduced by 1.3 of the original size.

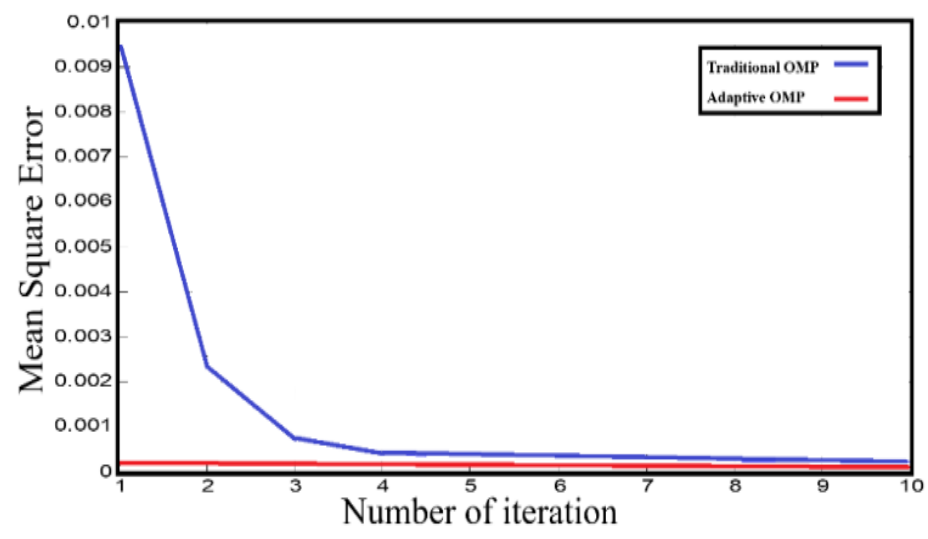

Figure 9. MSE of the classical and the adaptive OMP

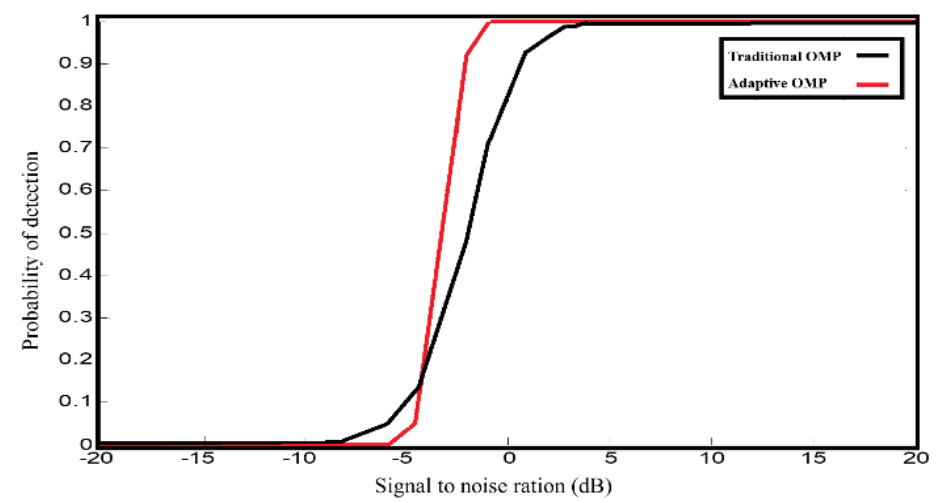

Figure 10. ROC curves for reconstructing image using the classical and the adaptive OMP algorithms

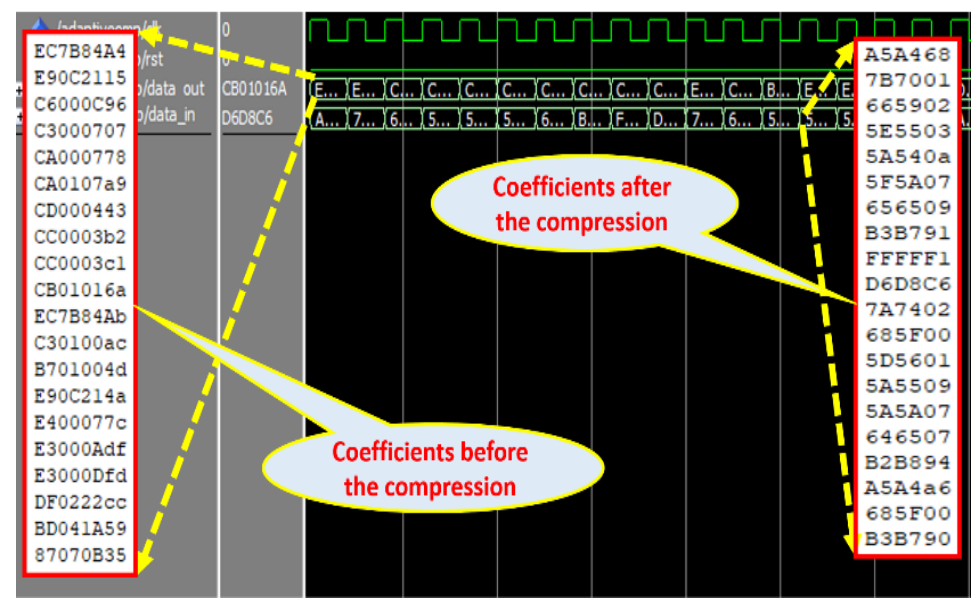

Figure 11. The modelsim simulation of the compression 


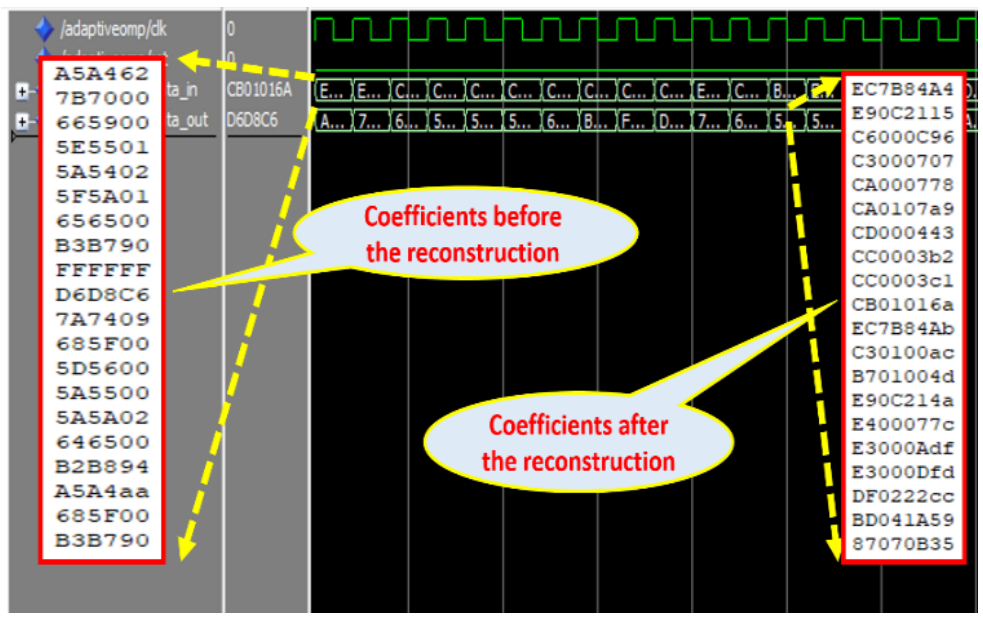

Figure 12. The modelsim simulation of the adptive OMP reconstruction

Figure 13 and 14 shows the ChipScope output after downloading the adaptive OMP project on the used kit. These Figures show the output at the real time and prove that the proposed algorithm can be used in the real life. This lap experimental is performed by using real SAR image to make the test environment as it is on the SAR onboard. The adaptive OMP gives more flexibility to update or debug the system. Table 1 highlights the device utilization summary for the classical OMP while Table 2 shows the summary for the adaptive OMP. From Table 1 and Table 2, the adaptive OMP consumed less hardware resourses and power consumption than the classical OMP. This reduction in hardware resourses and power consumption due to using certain threshold in adaptive OMP to reduce the numbers of iterarions and simplify the mathematical opertations which leads also to simplify the hardware implementation.

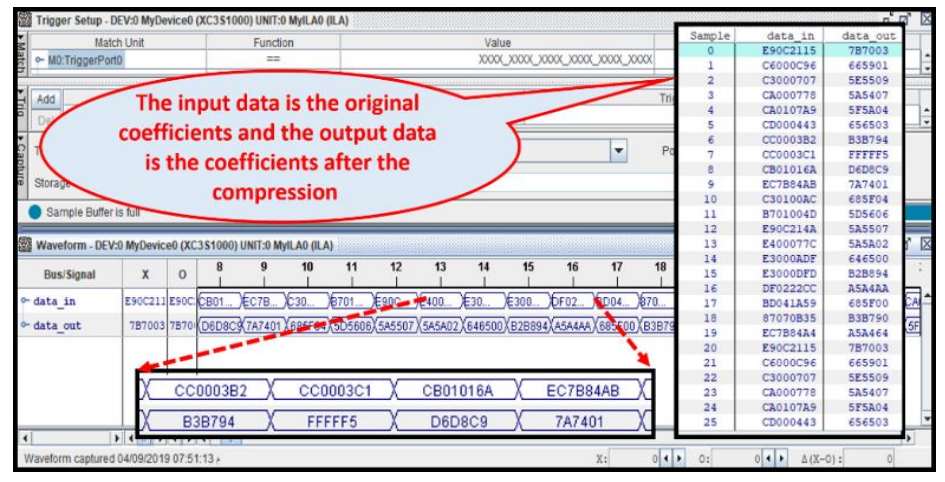

Figure 13. the chipscope signals in the compression case

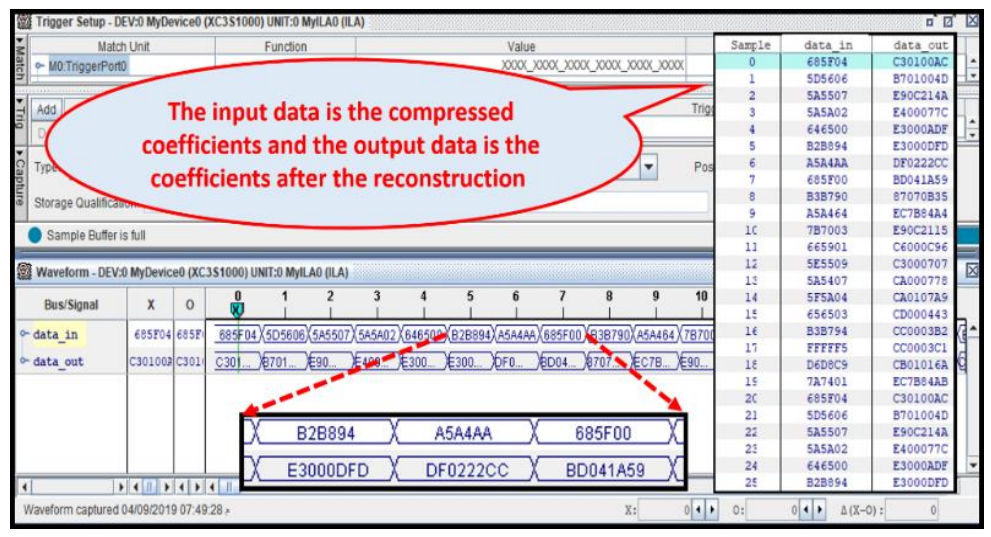

Figure 14. The chipscope signals in the reconstruction case 
Table 1. The device utilization summary for classical OMP

\begin{tabular}{cc}
\hline Logic Utilization & Used \\
\hline Number of Slice Flip Flops & 1235 \\
Number of 4 input LUTs & 320 \\
Number of occupied Slices & 2123 \\
Number of Slices containing only related & 1548 \\
logic & 2123 \\
Total Number of 4 input LUTs & 43 \\
Number of bonded IOBs & \\
\hline
\end{tabular}

Table 2. The device utilization summary for adaptive OMP

\begin{tabular}{cc}
\hline Logic Utilization & Used \\
\hline Number of Slice Flip Flops & 985 \\
Number of 4 input LUTs & 250 \\
Number of occupied Slices & 1578 \\
Number of Slices containing only related & 1456 \\
logic & \\
Total Number of 4 input LUTs & 1578 \\
Number of bonded IOBs & 31
\end{tabular}

\section{CONCLUSION}

OMP algorithm is efficiently used in SAR radar, however it has a high computional complexity in the image reconstruction process. To reduce its complexity and to improve its efficiency as well, amodification of this algorithm and its implementation using FPGA are proposed in this paper. The modification is mainly based on the threshold calculation method which used to reduce the hardware complexity in addition to the consumed processing time compared with the classical OMP algorithm. Also, a compressive sensing technique is used to make the OMP algorithm more suitable, significant, and more efficient in the images reconstruction of SAR systems. Reliability of the proposed algorithm is proved through its implementation using FPGA. Matlab simulation results and implementation show the effectiveness and low cost of the proposed algorithm compared to the classical OMP algorithm.

\section{REFERENCES}

[1] S.1. Kim, K. Koh, M. Lustig, S. Boyd, and D. Gorinevsky, "A metnod for large scale 11 -regulaized leat sqaures," IEEE Trans. Selected Topics Signal Processing, vol. 1, no. 4, pp. 606-617, Dec. 2007.

[2] N. Laska and R. G. Baraniuk, "Regime change: Bit-depth versus measurement-rate in compressive sensing," IEEE Trans. Signal Process., vol. 60, no. 7, pp. 3496-3505, 2012.

[3] Neshatpour, Katayoun, et al., "Architectural considerations for FPGA acceleration of Machine Learning Applications in MapReduce," International Conference on Embedded Computer Systems: Architectures, Modeling and Simulation (SAMOS), 2018.

[4] F. Salahdine and H. El Ghazi, "A Real Time Spectrum Scanning Technique based on Compressive Sensing for Cognitive Radio Networks," IEEE Annu. Ubiquitous Comput. Electron. Mob. Commun. Conf., pp. 1-6, 2017.

[5] Makrani, Hosein Mohammadi, "Storage and Memory Characterization of Data Intensive Workloads for Bare Metal Cloud." arXiv preprint arXiv: 1805.08332, 2018.

[6] G. Z. Karabulut, A. Yongacoglu, "Sparse cahnnel estimation using orthogonal matching pursuit algorithm," IEEE 60th Vehicular Technology Conference, 2004. VTC2004-Fall. 2004, 26-29 Sept. 2004.

[7] Yahan Wang; Huihui Bai; Yao Zhao, "Image Reconstruction from Patch Compressive Sensing Measurements," 2018 IEEE Fourth International Conference on Multimedia Big Data (BigMM), 2018.

[8] Sayadi, Hossein, et al., "Customized Machine Learning-Based Hardware-Assisted Malware Detection in Embedded Devices," IEEE TrustCom, 2018.

[9] S. Kunis, h. Rauhat, "Random sampling of sparse trigonometric polynomials II: Orthogonal matching pursuit versus basis pursuit," Applied and computational harmonic analysis journal, vol. 22, pp. 16-42, 2007.

[10] Vorapoj Patanavijit, "Computational scrutiny of image denoising method found on DBAMF under SPN surrounding," International Journal of Electrical and Computer Engineering (IJECE), vol. 10, no. 4, August 2020 (Part II).

[11] Ledya Novamizanti, Gelar Budiman, Elsa Nur Fitri Astuti, "Robust audio watermarking based on transform domain and SVD with compressive sampling framework," TELKOMNIKA (Telecommunication Computing Electronics and Control), vol. 18, no. 2, April 2020.

[12] Majid S. Naghmash, Nazar J. Alhyani, Ali M. Kadhim, "Optimization of image compression and ciphering based on EZW techniques," TELKOMNIKA (Telecommunication Computing Electronics and Control), vol. 18, no. 1, February 2020.

[13] Narayan Sharma; Rajoo Pandey, "Compressive Sensing and Recovery of Image Using Uniform Block Sparsity," 2018 3rd International Conference for Convergence in Technology (I2CT), pp. 1-5, 2018.

[14] H. B. Sharana basaveshwara; Santosh Herur, "Designing of Sensing Matrix for Compressive Sensing and Reconstruction," 2018 Second International Conference on Advances in Electronics, Computers and Communications (ICAECC), pp. 1-5, 2018.

[15] Andik Setyono, De Rosal Ignatius Moses Setiadi, "Image watermarking using discrete wavelet-tchebichef transform," Indonesian Journal of Electrical Engineering and Computer Science (IJEECS), vol. 16, no 3, December 2019.

[16] Eslam Ashraf, Ashraf A. M. Khalaf, Sara M. Hassan, "Pipelined Image Reconstruction of SAR Radar Based on Orthogonal Matching Pursuit using FPGA Implementation," International Journal of Recent Technology and Engineering, vol. 8, no. 5, January 2020. 
[17] Qiaoling Wu, Lin Ni, Delong He, "Improved Image Reconstruction Based on Block Compressed Sensing," IEEE International Conference, pp. 1964-1966, 2012.

[18] L. Ma, H. Bai, M. Zhang, Y. Zhao, "Edge-Based Adaptive Samplingfor Image Block Compressive Sensing," IEICE Transactions, vol.99-A, no. 11, pp. 2095-2098, 2016.

[19] Y. Arjoune, N. Kaabouch, H. E. Ghazi, and A. Tamtaoui, "Compressive sensing: Performance comparison of sparse recovery algorithms," 7th IEEE Ann. Comput. Commun. Workshop Conf., pp. 1-6, 2017.

[20] Pope Graeme, "Compressive sensinga summary of reconstruction algorithms," Masters Thesis, Department of Computer Science, Eidgen össische Technische Hochschule, Z ürich, February 2009.

[21] L. Anitori; W. van Rossum; M. Otten; A. Maleki; R. Baraniuk, "Compressive sensing radar: Simulation and experiments for target detection," 21st European Signal Processing Conferenc, 2013.

[22] Eslam Ashraf, Safa M.Gasser, Mohamed S. El-Mahallawy and Mohamed H.Abd ElAzeem, "Performance Enhancement of Wide-Band Radar Signals Using A New Adaptive CAMP Algorithm in Compressive Sensing," 5th International Conference on Electrical and Electronics Engineering, Istanbul, Turkey, May 2018.

[23] Jin Li ; Yao Fu ; Guoning Li ; Zilong Liu, "Remote Sensing Image Compression in Visible/Near-Infrared Range Using Heterogeneous Compressive Sensing," IEEE Journal of Selected Topics in Applied Earth Observations and Remote Sensing, pp. 4932-4938, 2018.

[24] E. 1. Candes, "The restricted isometry property and its implications for compressive sensing," C. R. l'Academie des SCiences, ser. I, no. 346, pp. 589-592, 2008.

[25] Anirban Bhowmik, Joydeep Dey, Arindam Sarkar, Sunil Karforma, "Computational intelligence based lossless regeneration (CILR) of blocked gingivitis intraoral image transportation," IAES International Journal of Artificial Intelligence (IJ-AI), vol. 8, no. 3, September 2019.

[26] Yasmine M. Tabra, Bayan Mahdi Sabbar, "FPGA implementation of new LM-SPIHT colored image compression with reduced complexity and low memory requirement compatible for 5G," International Journal of Reconfigurable and Embedded Systems (IJRES), vol. 8, no. 1, March 2019.

[27] Nafiseh Shahbazi, Aliazam Abbasfar and Mohammad Jabbarian- Jahromi, "Efficient two-dimensional compressive sensing in MIMO radar," EURASIP Journal on Advances in Signal Processing, pp. 1-15, December 2017.

[28] J. Tropp and A. Gilbert, "Signal recovery from random measurements via orthogonal matching pursuit," IEEE Transactions on Information Theory, vol. 53, no. 12, pp. 46-55, 2007.

[29] F. Salahdine and H. El Ghazi, "A Real Time Spectrum Scanning Technique based on Compressive Sensing for Cognitive Radio Networks," IEEE Annu. Ubiquitous Comput. Electron. Mob. Commun. Conf., pp. 1-6, 2017.

[30] Jost Tobias Springenberg, Alexey Dosovitskiy, ThomasBrox, and Martin Riedmiller, "Striving for simplicity: the all convolutional net," inICLR, 2015.

[31] Bhanumurthy, M. and Y. M. Reddy, "SAR Data Processing with Range Cell Migration,” International Journal of Engineering Science and Technology, pp. 1-11, 2011.

[32] Priyatharishini $\mathrm{m}$ and M. Nirmala Devi, "A compressive sensing algorithm for hardware trojan detection," International Journal of Electrical and Computer Engineering (IJECE), vol. 9, no. 5, October 2019 (Part II).

[33] Indrarini Dyah Irawati, Sugondo Hadiyoso, Yuli Sun Hariyani, "Multi-wavelet level comparison on compressive sensing for MRI image reconstruction," Bulletin of Electrical Engineering and Informatics (BEEI), vol. 9, no. 4, August 2020.

[34] Yilei Shi; Xiao Xiang Zhu; Richard Bamler, "Nonlocal Compressive Sensing-Based SAR Tomography," IEEE Transactions on Geoscience and Remote Sensing, vol. 57, no. 5, pp. 3015-3024, 2019.

[35] Hang Yin; Lei Wang, "Effect of Compressive Sensing Star Image on Attitude Determination," 2018 10th International Conference on Intelligent Human-Machine Systems and Cybernetics (IHMSC), pp. 8-11, 2018.

[36] Lakshminarayana M, Mrinal Sarvagya, "OFCS: Optimized Framework of Compressive Sensing for Medical Images in Bottleneck Network Condition," International Journal of Electrical and Computer Engineering (IJECE), vol. 8, no. 5, October 2018 (Part I).

[37] Lakshminarayana M, Mrinal Sarvagya, "MICCS: A Novel Framework for Medical Image Compression Using Compressive Sensing," International Journal of Electrical and Computer Engineering (IJECE), vol. 8, no. 5, October 2018 (Part I).

[38] Junichi Susaki; Masahiro Tsujino; Takuma Anahara, "Fusion of different frequency SAR images for DInSARbased land subsidence monitoring," 2017 IEEE International Geoscience and Remote Sensing Symposium (IGARSS), pp. 945-948, 2017.

\section{BIOGRAPHIES OF AUTHORS}

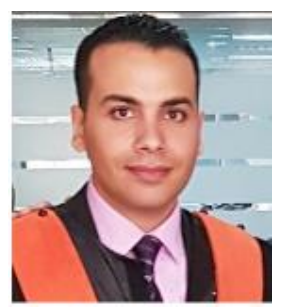

Eslam Ashraf received the B.Sc. degree of Electronics and Communications in 2009. He received master's degree in Electronics and Communications in 2018 from Arab Academy for Science, Technology and Maritime Transport.Currently, He is working towards $\mathrm{PhD}$ degree at Minia University at Electronics and Communications Department.

E-mail: eng.eslamashraff@gmail.com. 


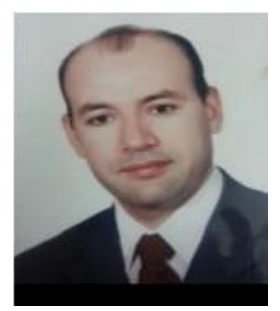

Ashraf A. M. Khalaf received his B.Sc. and M.Sc. degrees in electrical engineering from Minia University, Egypt, in 1989 and 1994 respectively. He received his Ph.D. in electrical engineering from Graduate School of Natural Science and Technology, Kanazawa University, Japan, in March 2000. He is currently associate professor at electronics and communications engineering Department, Faculty of Eng, Minia University, Egypt. E-mail: ashkhalaf@yahoo.com.

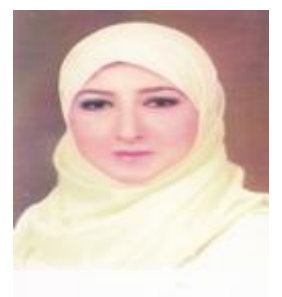

Sara M. Hassan received the B.Sc. degree in Electrical Engineering from Modern academy for engineering and technology, Cairo, Egypt, in 2007, the M. Sc. degree from Ain Shams University, Cairo, Egypt, in 2013, and the Ph.D. from Ain Shams University, Cairo, Egypt, in 2017. She is currently a staff member at Modern academy for engineering and technology, Cairo, Egypt. E-mail: Sara.Hassan@eng.modern-academy.edu.eg. 